\title{
Survival with AGS-003, an autologous dendritic cell-based immunotherapy, in combination with sunitinib in unfavorable risk patients with advanced renal cell carcinoma (RCC): Phase 2 study results
}

Asim Amin ${ }^{1 *}$, Arkadiusz Z Dudek ${ }^{2}$, Theodore F Logan ${ }^{3}$, Raymond S Lance ${ }^{4}$, Jeffrey M Holzbeierlein ${ }^{5}$, Jennifer J Knox ${ }^{6}$, Viraj A Master ${ }^{7}$, Sumanta K Pal ${ }^{8}$, Wilson H Miller Jr ${ }^{9}$, Lawrence I Karsh ${ }^{10}$, Irina Y Tcherepanova ${ }^{11}$, Mark A DeBenedette ${ }^{11}$, W Lee Williams $^{11}$, Douglas C Plessinger ${ }^{11}$, Charles A Nicolette ${ }^{11}$ and Robert A Figlin ${ }^{12^{*}}$

\begin{abstract}
Background: AGS-003 is an autologous immunotherapy prepared from fully matured and optimized monocyte-derived dendritic cells, which are co-electroporated with amplified tumor RNA plus synthetic CD40L RNA. AGS-003 was evaluated in combination with sunitinib in an open label phase 2 study in intermediate and poor risk, treatment naïve patients with metastatic clear cell renal cell carcinoma (mRCC).

Methods: Twenty-one intermediate and poor risk patients were treated continuously with sunitinib (4 weeks on, 2 weeks off per 6 week cycle). After completion of the first cycle of sunitinib, patients were treated with AGS-003 every 3 weeks for 5 doses, then every 12 weeks until progression or end of study. The primary endpoint was to determine the complete response rate. Secondary endpoints included clinical benefit, safety, progression free survival (PFS) and overall survival (OS). Immunologic response was also monitored.
\end{abstract}

Results: Thirteen patients (62\%) experienced clinical benefit (9 partial responses, 4 with stable disease); however there were no complete responses in this group of intermediate and poor risk mRCC patients and enrollment was terminated early. Median PFS from registration was 11.2 months ( $95 \% \mathrm{Cl} 6.0,19.4)$ and the median OS from registration was 30.2 months $(95 \%$ Cl 9.4, 57.1) for all patients. Seven (33\%) patients survived for at least 4.5 years, while five (24\%) survived for more than 5 years, including 2 patients who remain progression-free with durable responses for more than 5 years at the time of this report. AGS-003 was well tolerated with only mild injection-site reactions. The most common adverse events were related to expected toxicity from sunitinib therapy. In patients who had sequential samples available for immune monitoring, the magnitude of the increase in the absolute number of $C D 8^{+} C D 28^{+} \mathrm{CD}_{45} \mathrm{RA}^{-}$ effector/memory T cells (CTLs) after 5 doses of AGS-003 relative to baseline, correlated with overall survival.

Conclusions: AGS-003 in combination with sunitinib was well tolerated and yielded supportive immunologic responses coupled with extension of median and long-term survival in an unselected, intermediate and poor risk prognosis mRCC population.

Clinical Trial Registry: \#NCT00678119

Keywords: Immunotherapy, Dendritic cell, RCC, AGS-003, Sunitinib

\footnotetext{
*Correspondence: Asim.Amin@carolinashealthcare.org; robert.figlin@cshs.org

'Levine Cancer Institute, Charlotte, NC, USA

${ }^{12}$ Cedars-Sinai Medical Center, Los Angeles, CA, USA

Full list of author information is available at the end of the article
} 


\section{Background}

Renal cell carcinoma (RCC) is an immunologically responsive tumor and until recently, the mainstay for systemic therapy for advanced metastatic RCC was cytokine-based immunotherapy with interleukin 2 (IL-2) and interferon alpha (IFN- $\alpha$ ). During the past decade, identification of critical cellular growth factor pathways in RCC has enabled development of drugs targeting the vascular endothelial growth (VEGF) pathway and the mammalian target of rapamycin (mTOR) complex. Approved VEGF tyrosine kinase inhibitors (TKIs) and mTOR inhibitors have shown clinically relevant benefit in phase 3 trials [1]. Although durable responses are rarely seen with these targeted agents [1-3], they can be used for a broader spectrum of patients than cytokines and the clinical benefit as evidenced by improvements in both progression-free and overall survival, primarily in patients with favorable and intermediate risk profiles, has positively impacted the natural history of metastatic RCC (mRCC) [4].

In contrast to the VEGF and mTOR inhibitors, durable and complete responses have been observed with high dose (HD) IL-2, indicating the importance of immune modulation in this disease process [5]. While $25 \%$ may show response, only $5-8 \%$ of patients experience durable complete responses. HD IL-2 is, however, associated with significant toxicity, which has limited this modality to be delivered at specialized centers in a highly selected and limited population of mRCC patients with a high level of cardiopulmonary fitness [6]. Immune modulation, if it can be delivered with low toxicity and be applicable to a broader mRCC population, would be a desirable therapeutic approach to pursue in combination with standard targeted therapy.

Dendritic cells (DCs) are a powerful tool for stimulating cell-mediated immunity by efficient presentation of antigen to both $\mathrm{CD}^{+}$and $\mathrm{CD} 8^{+}$T cells [7,8]. AGS-003 is an autologous immunotherapy approach which provides the critical signals required to generate a patient and tumor specific adaptive immune response. AGS-003 is prepared ex vivo from matured monocyte-derived DCs co-electroporated with the patient's amplified tumor RNA and synthetic CD40L RNA [9-12]. When administered by intradermal injection, these optimized, RNA-loaded mature DCs are capable of presenting the relevant patient-specific tumor antigens, via MHC-Class I presentation, to $\mathrm{T}$ cells in the draining lymph node basin. Additionally, CD40 ligation optimizes $\mathrm{CD}^{+} \mathrm{T}$ cell induction through production of IL-12 $[11,12]$. AGS-003 has previously been evaluated as a monotherapy [13] in newly diagnosed, intermediate and poor risk, synchronous mRCC patients and was tolerated well with no grade 3 or 4 adverse events. In a predominantly poor risk population, median PFS was 5.5 months and median overall survival (OS) was 15.7 months, with $23 \%$ of patients surviving for 3.5 to 8 plus years, despite limited use of any subsequent systemic therapy, including targeted therapy (T. Logan, A. Amin, V. Cohen, et al. A Phase 1/2 Study of AGS-003, a personalized immunotherapeutic evaluated in newly diagnosed metastatic renal cell carcinoma subjects; In preparation). The use of autologous tumor RNA for broad tumor antigen presentation by autologous, mature DCs holds promise as a fully personalized, patientspecific immunotherapeutic product as it minimizes the risk of mutant clonal escape with presentation of multiple target antigens from the autologous tumor sample [14-17].

Sunitinib is the standard of care for first-line treatment of mRCC. It is particularly attractive for combination therapy with a novel immunotherapeutic approach. Sunitinib has been shown to elicit a positive modulatory effect on the immune system through suppression of myeloidderived suppressor cells (MDSCs) and T regulatory (Treg) cells $[18,19]$. Tumors are infiltrated with Treg cells and MDSCs that actively inhibit T-cell responses and contribute to altered immune surveillance in RCC [20,21]. While targeted therapies such as sunitinib have yielded improved efficacy over the past decade, durable remissions and long-term survival are rare, particularly in newly diagnosed, intermediate and poor risk metastatic RCC (mRCC) patients. A recent analysis by the International mRCC Database Consortium (IMDC) indicates newly diagnosed, intermediate and poor risk mRCC patients who present with the time from diagnosis to initiation of treatment less than 1 year risk factor $(\mathrm{DxTx}<1 \mathrm{y})$, have an expected median progression-free survival (PFS) of 5.6 months and median overall survival (OS) of 14.7 months, despite treatment with standard targeted therapy [22]. When treated with sunitinib alone, the expected percentage of intermediate and poor risk mRCC patients surviving greater than 30 months is 13\% [23].

The Phase 2 study was designed to assess the efficacy and safety of AGS-003 in combination with sunitinib in patients with newly diagnosed, intermediate and poor risk, synchronous mRCC after nephrectomy.

\section{Results}

\section{Patients}

Of 25 enrolled patients who underwent leukapheresis, three patients were withdrawn prior to study treatment, either due to rapid disease progression $(n=1)$, uncontrolled hypertension $(n=1)$ or withdrawal of consent $(n=1)$ (Figure 1). Twenty two patients entered the induction phase and received sunitinib and at least one dose of AGS-003, including one patient who had enrolled in a prior AGS-003 study. This rollover patient was excluded from efficacy evaluations and included for safety evaluation only.

Demographics and tumor characteristics for the efficacy population $(\mathrm{n}=21)$ are shown in Table 1 . Median patient age was 56 years (range 22-68), all treated patients had clear cell mRCC, and the majority were Fuhrman 

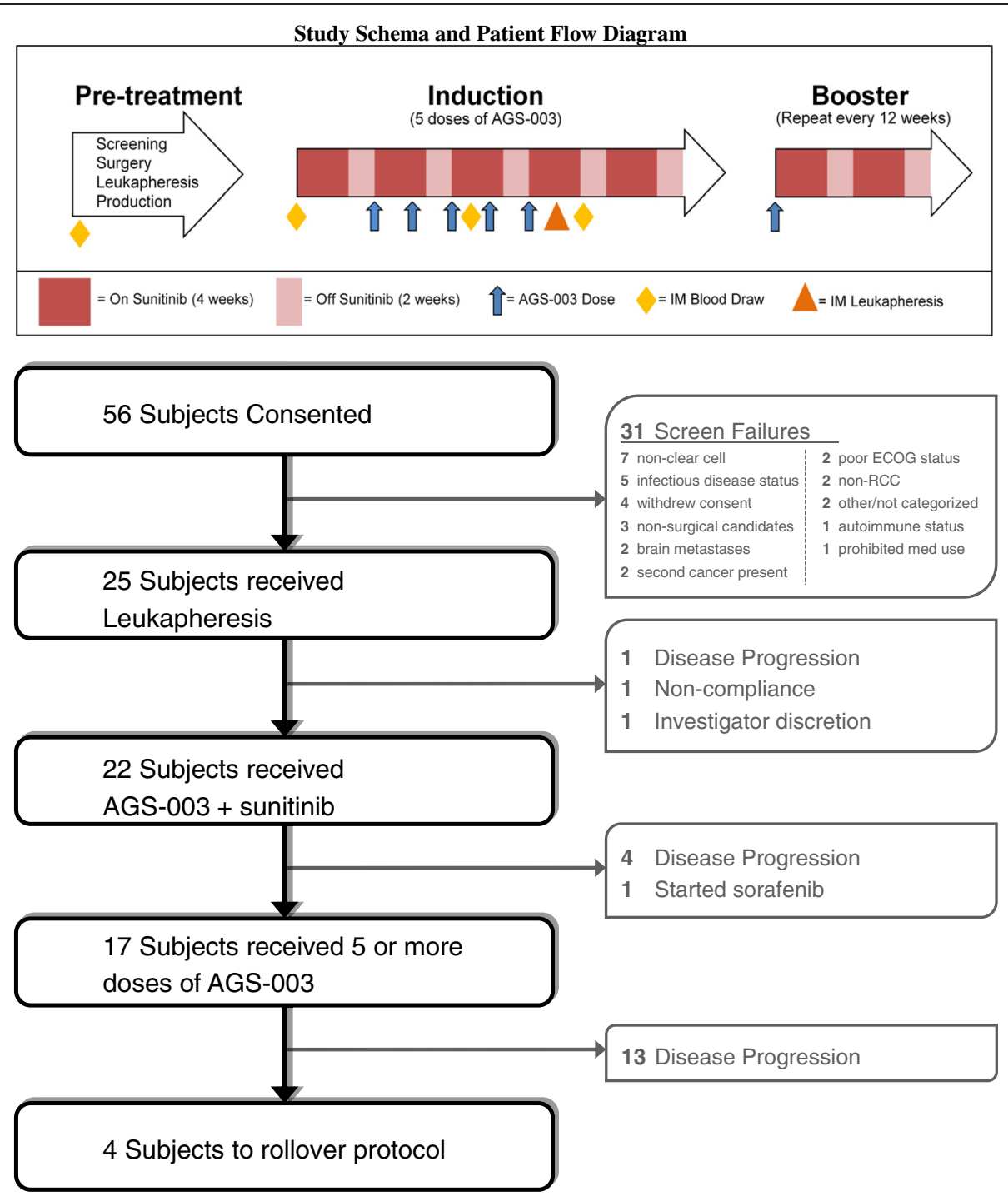

Figure 1 Study Schema and Patient Flow Diagram.

grade 3 or $4(76 \%, 16 / 21)$ and were T3 or T4 $(72 \%, 15 / 21)$. All study patients presented with synchronous, measurable metastatic disease and had a time from diagnosis to treatment of less than 1 year (mean $=10.8 \pm 6.41$ weeks). Patients were classified as intermediate $(71 \%)$ or poor (29\%) risk according to Memorial Sloan Kettering Cancer Center (MSKCC) [24] and intermediate $(52 \%)$ or poor (48\%) risk according to Heng criteria [25].

\section{AGS-003 Production, Characteristics, and Patient Exposure}

The median time from leukapheresis to production and administration of the first AGS-003 dose was 7 weeks. The median time from nephrectomy/metastasectomy to first dose was 11 weeks. The median number of AGS003 doses administered was 6 (2-15). Nine patients (43\%) received eight or more AGS-003 doses. Eight patients $(38 \%)$ had sunitinib dose delays or dose reductions during the trial, including one patient who discontinued sunitinib while on study. Overall, 10 (48\%) patients received subsequent therapy after progression, including TKIs $(\mathrm{n}=6)$, chemotherapy $(\mathrm{n}=4)$, mTOR inhibitor $(\mathrm{n}=2)$, cytokine therapy $(\mathrm{n}=1)$ and investigational immunotherapy $(\mathrm{n}=1)$.

\section{Clinical Responses Tumor Responses}

The best overall tumor response per RECIST, after at least one 6-week cycle of sunitinib, are shown in Table 2. No complete responses (CRs) were observed. Overall, $43 \%(9 / 21)$ of patients experienced a partial response (PR) as their best response (2 PRs were observed following the initial 6-week cycle of sunitinib). In addition, 4 PRs were observed during induction with AGS-003 plus sunitinib, while 3 additional PRs developed during the 
Table 1 Demographics and Patient RCC Characteristics

\begin{tabular}{|c|c|}
\hline Characteristics & Patients $\mathrm{N}=\mathbf{2 1}$ \\
\hline Age, median years (range) & $56.0(22-68)$ \\
\hline \multicolumn{2}{|l|}{ Sex, n (\%) } \\
\hline Male & $16(76)$ \\
\hline Female & $5(24)$ \\
\hline \multicolumn{2}{|l|}{ Race, n (\%) } \\
\hline Caucasian & $19(91)$ \\
\hline Black & $1(5)$ \\
\hline Asian & $1(5)$ \\
\hline $\begin{array}{l}\text { Time from diagnosis to } 1^{\text {st }} \\
\text { sunitinib dose, mean weeks (SD) }\end{array}$ & $10.8(6.41)$ \\
\hline \multicolumn{2}{|l|}{ ECOG performance status, $n$ (\%) } \\
\hline 0 & $15(71)$ \\
\hline 1 & $6(29)$ \\
\hline \multicolumn{2}{|l|}{ MSKCC risk [23], n (\%) } \\
\hline Favorable (0 risk factors) & 0 \\
\hline Intermediate (1-2 risk factors) & $15(71)$ \\
\hline Poor ( $\geq 3$ risk factors) & $6(29)$ \\
\hline \multicolumn{2}{|l|}{ Heng risk [24], n (\%) } \\
\hline Favorable (0 risk factors) & 0 \\
\hline Intermediate (1-2 risk factors) & $11(52)$ \\
\hline Poor ( $\geq 3$ risk factors) & $10(48)$ \\
\hline \multicolumn{2}{|l|}{ Fuhrman nuclear grade, $\mathbf{n}(\%)$} \\
\hline Grade 2 & $5(24)$ \\
\hline Grade 3 & $9(43)$ \\
\hline Grade 4 & $7(33)$ \\
\hline \multicolumn{2}{|l|}{ Tumor Size, n (\%) } \\
\hline TX & $2(10)$ \\
\hline $\mathrm{T} 1$ & $3(14)$ \\
\hline T2 & $1(5)$ \\
\hline T3 & $13(62)$ \\
\hline T4 & $2(10)$ \\
\hline \multicolumn{2}{|c|}{ Regional Lymph Nodes Staging, n (\%) } \\
\hline NX & $10(48)$ \\
\hline No & $6(29)$ \\
\hline N1 & $1(5)$ \\
\hline N2 & $4(19)$ \\
\hline \multicolumn{2}{|l|}{ Distant Metastasis Staging, $\mathbf{n}(\%)$} \\
\hline M1 & $21(100)$ \\
\hline
\end{tabular}

ECOG = Eastern Cooperative Oncology Group MSKCC = Memorial Sloan Kettering Cancer Center; $\mathrm{n}=$ number of patients; $\mathrm{SD}=$ standard deviation.

prolonged booster phase, more than 1 year after initiating combined treatment. Two of these patients continue on study treatment for more than 5 years at the time of this report. Additionally, 4 patients experienced stable
Table 2 Tumor response data

\begin{tabular}{|c|c|c|c|c|}
\hline \multirow[t]{2}{*}{ Best overall response* } & PR & SD & $\mathrm{PR}+\mathrm{SD}$ & PD \\
\hline & \multicolumn{4}{|l|}{$\mathrm{N}(\%)$} \\
\hline All patients $(n=21)$ & $5(24)$ & $8(38)$ & $13(62)$ & $8(38)$ \\
\hline MSKCC intermediate risk $(n=15)$ & $4(19)$ & $7(33)$ & $11(52)$ & $4(19)$ \\
\hline MSKCC poor risk $(n=6)$ & $1(5)$ & $1(5)$ & $2(10)$ & $4(19)$ \\
\hline Heng intermediate risk $(n=11)$ & $4(19)$ & $5(24)$ & $9(43)$ & $2(10)$ \\
\hline Heng poor risk $(n=10)$ & $1(5)$ & $3(14)$ & $4(19)$ & $6(29)$ \\
\hline
\end{tabular}

disease. The overall clinical benefit rate for the combination $(\mathrm{CR}+\mathrm{PR}+$ stable disease $[\mathrm{SD}])$ was $62 \%(13 / 21)$.

\section{Progression-Free Survival}

Median PFS (95\% CI) was 11.2 months (6.0, 19.4). Based upon the Heng risk criteria, the median PFS was 5.8 (4.3, 11.2) months for poor risk and 19.4 (7.2, 38.1) months for intermediate risk patients. The Kaplan-Meier PFS estimates for all patients and by Heng risk group are shown in Table 3.

\section{Overall Survival}

Median OS (95\% CI) for all patients was $30.2(9.4,57.1)$ months. Based on the Heng risk criteria, the estimated median OS was 61.9 months $(16.3, \mathrm{NE})$ for intermediate risk and $9.1(5.3,30.2)$ months for the poor risk patients. The Kaplan-Meier OS estimates for all patients and by Heng risk group are shown in Figure 2.

Overall, $52 \%(11 / 21)$ of patients experienced long-term survival $>30$ months. At the time of this report, 7 (33\%) patients are alive more than 4.5 years from study registration, while 5 (24\%) are alive for more than 5 years, including two intermediate risk patients who remain progression-free and continue to receive booster AGS003 dosing and reduced-dose sunitinib on a separate protocol.

\section{Immune Responses}

Fourteen of 21 patients received 5 doses of AGS-003, underwent a subsequent leukapheresis, and had evaluable samples for immunologic analyses. Preclinical studies demonstrated that AGS-003 administration results in the generation of $\mathrm{CD}^{+} \mathrm{CD} 28^{+} \mathrm{CD} 45 \mathrm{RA}^{-}$effector memory CTLs. Previous published work revealed that in vitro priming with post matured CD40L RNA electroporated DCs encoding specific target antigen expanded multifunctional antigen specific CTLs exhibiting an effector memory phenotype defined by the expression of CD28 and negative for CD45RA expression. Therefore the primary immune monitoring end point analysis focused on identifying increases in the numbers of functional CTLs 
Table 3 Kaplan-Meier estimates of progression free survival for all patients and for patients in Heng intermediate and poor risk factor groups*

\begin{tabular}{|c|c|c|c|c|c|c|c|c|c|c|c|c|c|c|}
\hline \multirow{2}{*}{$\begin{array}{l}\text { Patient group } \\
\text { Month }\end{array}$} & \multicolumn{13}{|c|}{ Number of patients at risk } & \multirow{2}{*}{$\begin{array}{l}\text { Median time to } \\
\text { progression or death } \\
\text { Months }(95 \% \mathrm{Cl})\end{array}$} \\
\hline & 0 & 3 & 6 & 9 & 12 & 15 & 18 & 21 & 24 & 27 & 30 & 33 & 36 & \\
\hline All patients $(n=21)$ & 21 & 21 & 15 & 12 & 10 & 8 & 7 & 6 & 4 & 4 & 3 & 1 & 1 & $11.2(6,19.4)$ \\
\hline Heng intermediate risk group $(n=11)$ & 11 & 11 & 11 & 9 & 8 & 6 & 6 & 5 & 4 & 4 & 3 & 1 & 1 & $19.4(7.2,38.1)$ \\
\hline Heng poor risk group $(n=10)$ & 10 & 10 & 4 & 3 & 2 & 2 & 1 & 1 & 0 & 0 & 0 & 0 & 0 & $5.8(4.3,11.2)$ \\
\hline
\end{tabular}

*Similar results were obtained for MSKCC intermediate (14.9 months) and poor (5.7 months) risk factor groups.

$\mathrm{Cl}=$ confidence interval.

expressing CD28 in the absence of CD45RA after administration of AGS-003 [11,12]. A CTL was considered functional if it expressed any of the functional markers defined by cytokine secretion (IFN-g, TNF-a, and IL-2), lytic function (CD107a), or proliferated (Brdu). Figure 3 outlines the gating strategy to detect functional CD28+/CD45RA- CTLs. Functional CD28+/CD45RACTLs were quantitated prior to systemic therapy (postnephrectomy prior to sunitinib) and after 5 doses of AGS-003 in combination with sunitinib. The baseline level for each patient was determined by averaging the absolute number of functional CD28+/CD45RA-CTLs/ $\mathrm{ml}$ after in vitro stimulation for the two baseline samples, one pre- and one post-nephrectomy, both prior to AGS-003 administration. The absolute numbers of functional CD28+/CD45RA-CTLs were determined after the $5^{\text {th }}$ dose of AGS-003. Neither the baseline response prior to AGS-003 administration (Figure 4A) nor the response after the $5^{\text {th }}$ dose of AGS-003 (Figure 4B) correlated with overall survival (Figure 4D). However after the $5^{\text {th }}$ dose, 10 of 14 (71\%) patients analyzed displayed an increase in the number of functional CD28+/CD45RA-
CTLs over baseline (Figure 4C). Furthermore, this change in the magnitude of the response showed a statistically significant correlation with the duration of survival (nonparametric bivariate analysis, Spearman's $\rho=0.8 ; \mathrm{p}<$ 0.002). Therefore the detection of newly generated CTLs are most likely to be tumor antigen-specific following stimulation with autologous DCs electroporated with autologous amplified tumor RNA. Notably, one patient exhibited prolonged survival in the absence of a detectable increase in the absolute number of CTLs. Interestingly, this patient had significant numbers of CTLs at baseline, prior to AGS-003 initiation.

\section{Safety}

Treatment emergent adverse events occurring in five or more patients are listed in Table 4 . Table 4 also shows the relationship of adverse events to study treatments (AGS-003, sunitinib or combination). All patients reported at least one event. There were no grade 4 toxicities reported, and no patient withdrew from the study due to an adverse event. Nine patients had grade 3 events attributed to sunitinib treatment; none of these were attributed

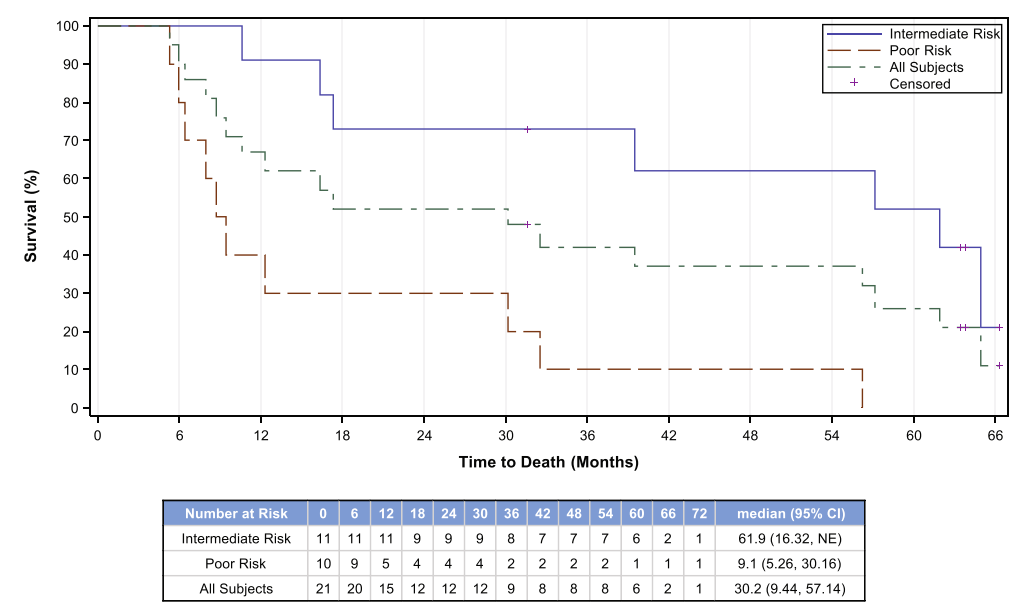

Figure 2 Overall survival. Kaplan-Meier estimates of overall survival (months) for all patients and by patients in Heng intermediate and poor risk factor groups. Similar results were obtained for MSKCC intermediate (39.5 months) and poor (7.9 months) risk factor group. 


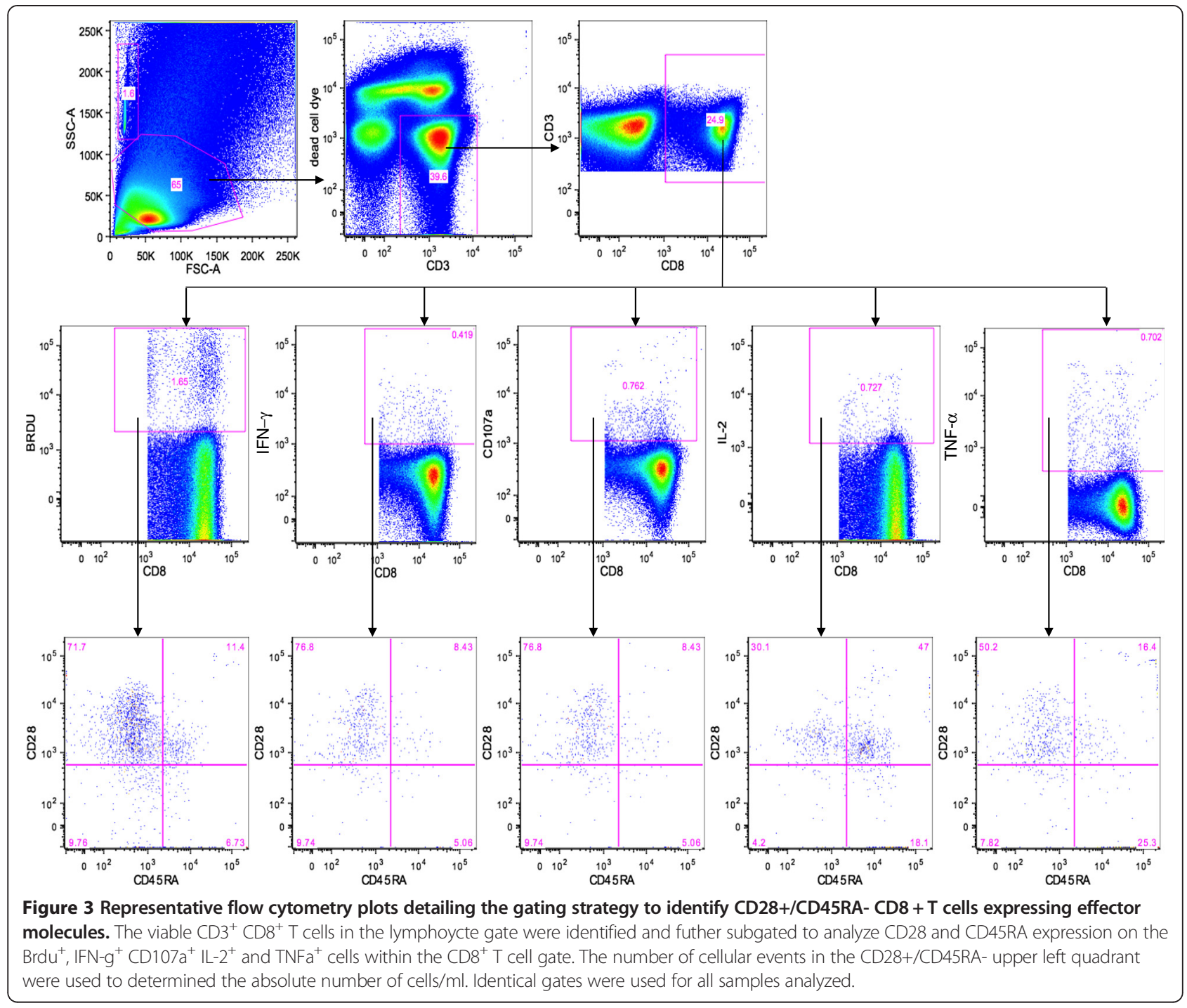

to AGS-003 treatment. There were seven treatment emergent serious adverse events reported (one case each of bradycardia, constipation, diarrhea, pneumonia, dehydration, hypocalcemia and urinary retention), that resolved without sequelae with standard management. No grade 3 or serious adverse event was considered related to AGS003. There were no treatment related deaths.

The most frequent AGS-003-related events were mild (grade 1) injection-site reactions, including erythema (7/ 22 patients), induration $(5 / 22)$, swelling $(4 / 22)$ pain $(3 /$ $22)$ and pruritus $(3 / 22)$. No adverse events unique to the combination of AGS-003 plus sunitinib were reported.

Adverse events reported within 24 hours of a leukapheresis procedure occurred in eleven patients, including vomiting in two patients (one of whom also had nausea and decreased weight), four patients with pain, three patients with fatigue, one patient with constipation and nausea, and one patient with muscle spasms and oral paresthesia. All were mild to moderate in severity and did not preclude further participation nor require hospitalization.

No evidence of emergent autoimmunity was noted per laboratory assessments for auto-immune markers. There were no clinically relevant outcomes for hematology, biochemistry, and urinalysis laboratory assessments, physical examinations, vital signs, or ECGs.

\section{Discussion}

During the past decade, VEGF-targeted therapies have become standard treatment for advanced RCC. While targeted therapies have yielded improved efficacy, durable remissions, CRs $(<1 \%)$ and long-term survival ( $>30$ months) are rare, particularly in intermediate and poor risk metastatic RCC (mRCC) patients [25,26]. A recent analysis by the International mRCC Database Consortium (IMDC) revealed that newly diagnosed, intermediate 

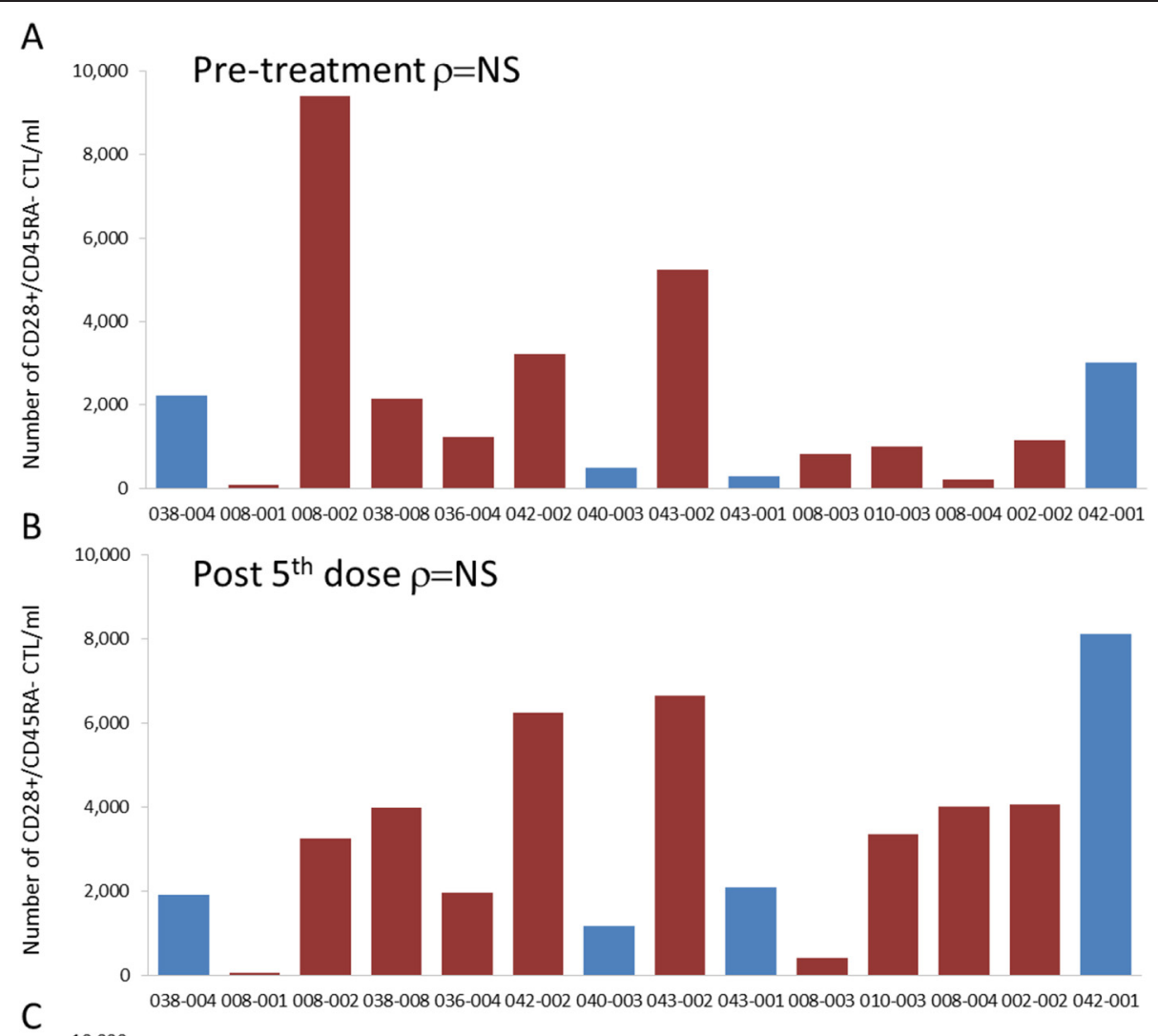

C ${ }^{10,000}$ Change from pre-treatment to Post $5^{\text {th }}$ dose $\rho=<0.002$

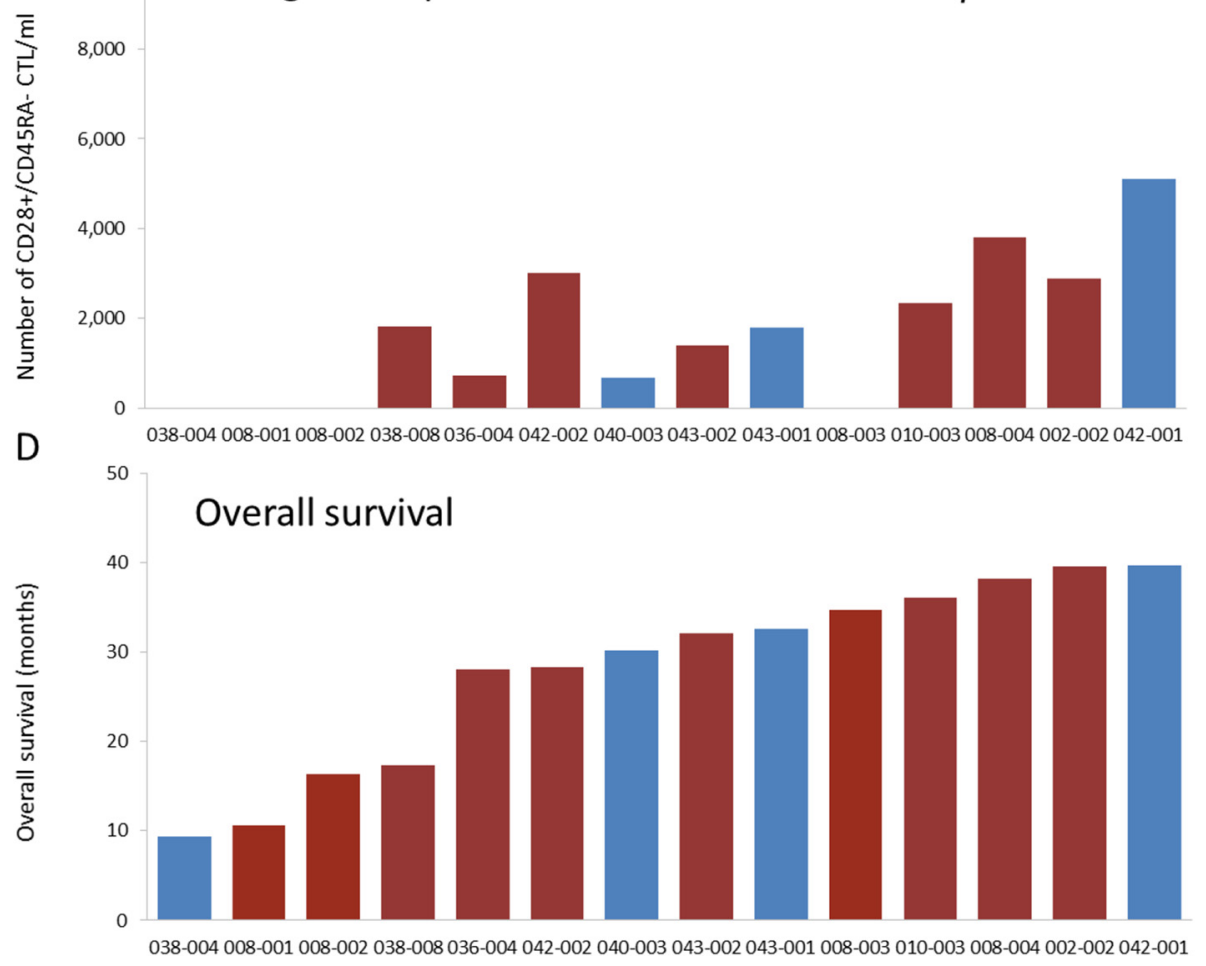

Figure 4 (See legend on next page.) 
(See figure on previous page.)

Figure 4 Measurement of AGS-003 induced multifunctional CD8 ${ }^{+} \mathrm{CD} 28^{+} \mathrm{CD} 45 \mathrm{RA} \mathrm{C}^{-} \mathrm{CTL}$ and correlation with overall survival. $\mathrm{Abs}$ (ute number of CD28+/CD45RA- CTLs in response to DCs electroporated with autologous amplified RCC tumor RNA at baseline (A) and after 5 doses of AGS-003 (B). Increase in the number of CD28+/CD45RA- CTL from baseline to post $5^{\text {th }}$ dose (C). Patients are listed by in increasing order of overall survival (D). Blue bars ( ) identify intermediate risk patients and red bars ( $)$ identify poor risk patients. The correlation between the absolute number of CD28+/CD45RA- CTLs and overall survival was statistically significant by nonparametric bivariate analysis, Spearman's $\rho=0.8 ; p<0.002$.

and poor risk mRCC patients who present with the time from diagnosis to initiation of treatment less than 1 year risk factor $(\mathrm{DxTx}<1 \mathrm{y})$, have an expected median PFS of 5.6 months and median OS of 14.7 months, despite treatment with standard, sequential targeted therapy [22]. Attempts to improve outcome using combinations of targeted agents including TKIs, mTORs, plus interferon have been limited due to severe and/or overlapping toxicity [27-30]. While immune modulation with IL-2 has shown durable responses, it is limited to a select population of patients. Therefore, there is a need to identify novel immunomodulatory therapies for mRCC.

The rationale to combine sunitinib with AGS-003, a fully personalized immunotherapy, was based upon the proven antitumor activity of sunitinib and its potential to attenuate the immune suppression in the tumor microenvironment observed in advanced $\operatorname{RCC}[18,19]$. In this study, AGS-003 was evaluated in combination with sunitinib for the treatment of newly diagnosed mRCC patients with intermediate or poor risk as defined by either MSKCC or Heng criteria. All patients presented with the DxTx $<1$ yr risk factor and the majority presented with at least 2, 3 or 4 risk factors. When this study was initially designed, the primary objective was to determine whether the addition of AGS-003 to sunitinib could achieve a $20 \%$ complete remission rate. As TKI data matured, it became evident that sunitinib was rarely if ever associated with CRs in advanced RCC, even in studies where approximately one-third of subjects entered with favorable risk disease $[26,31,32]$. Based on emerging data and insights during study conduct, it became evident that the primary endpoint was inappropriate for this population. Therefore, further enrollment was stopped. An independent safety monitoring board established at study start continued to monitor safety for the duration of the study. Patients already enrolled continued treatment and follow-up per

Table 4 Treatment emergent adverse events occurring in 5 or more patients and relationships* to study drug treatment

\begin{tabular}{|c|c|c|c|c|}
\hline \multirow[t]{2}{*}{ Adverse event } & \multicolumn{4}{|c|}{ Treatment-Emergent adverse events $\mathrm{N}=\mathbf{2 2}$ Number of patients (\%) } \\
\hline & All & AGS-003 related ${ }^{* *}$ & Sunitinib related & Combination related \\
\hline Any & $22(100)$ & $17(77)$ & $22(100)$ & $12(55)$ \\
\hline Diarrhea & $13(59)$ & $3(14)$ & $11(50)$ & $3(14)$ \\
\hline Fatigue & $13(59)$ & $3(14)$ & $12(55)$ & $2(9)$ \\
\hline Nausea & $12(55)$ & $2(9)$ & $11(50)$ & $2(9)$ \\
\hline Rash & $10(46)$ & $4(18)$ & $9(41)$ & $5(23)$ \\
\hline Decreased weight & $9(41)$ & $1(5)$ & $5(23)$ & $1(5)$ \\
\hline Headache & $8(36)$ & $2(9)$ & $4(18)$ & $2(9)$ \\
\hline Injection-site erythema & $8(36)$ & $7(32)$ & 0 & 0 \\
\hline Peripheral edema & $8(36)$ & 0 & $4(18)$ & 0 \\
\hline Dyspnea & $7(32)$ & 0 & $1(5)$ & 0 \\
\hline Vomiting & $7(32)$ & 0 & $7(32)$ & 0 \\
\hline Anorexia & $6(27)$ & 0 & $5(23)$ & $1(5)$ \\
\hline Hypertension & $6(27)$ & $1(5)$ & $6(27)$ & $1(5)$ \\
\hline Hypothyroidism & $6(27)$ & 0 & $5(23)$ & $1(5)$ \\
\hline Hand-foot syndrome & $6(27)$ & 0 & $6(27)$ & 0 \\
\hline Constipation & $5(23)$ & 0 & $2(9)$ & 0 \\
\hline Dehydration & $5(23)$ & 0 & $2(9)$ & 0 \\
\hline Dysgeusia & $5(23)$ & $1(5)$ & $5(23)$ & $1(5)$ \\
\hline Injection-site induration & $5(23)$ & $5(23)$ & 0 & $1(5)$ \\
\hline
\end{tabular}

*Events described as possible, probably or definitely related to study drug.

${ }^{* *}$ All AGS-003 related events were Grade 1 or 2 in severity. 
protocol to assess secondary endpoints such as PFS, OS and safety. These endpoints were deemed to be much more instructive for future AGS-003 development efforts in similar, newly diagnosed intermediate and poor risk mRCC patients.

In addition to limited expectations for durable complete remissions with targeted therapies such as sunitinib, these treatments also appear to exert both cytotoxic and cytostatic effects which complicate response assessment, particularly when combined with immunotherapy. RECIST-based criteria used traditionally for evaluating response to cytotoxic agents may therefore not be adequate or helpful to evaluate clinically relevant outcomes with targeted therapies alone, since they may induce necrosis with minimal change in size with conventional imaging [33]. Yet another layer of complexity regarding response evaluation is added by the immunotherapy component used in combination with sunitinib in this study. Observations with immunotherapy in patients with metastatic melanoma treated with agents such as ipilimumab, led to the development of immune response criteria that showed disease stability translates into clinical benefit. Delayed responses were noted even after initial increases in tumor burden [34]. Therefore, disease stabilization and overall survival may be more appropriate measures of clinical benefit with new immunotherapeutic modalities such as AGS-003. This is further supported by the proportion of melanoma patients treated with ipilimumab demonstrating durable, long term overall survival at a follow-up of 5 to 6 years [35].

In this study, the median PFS for all subjects was 11.2 months and the median OS was 30.2 months, which represents a near doubling of expected survival in this group of patients. The median OS was estimated to be more than 5 years at 61.9 months for the 11 patients classified as Heng intermediate risk (1-2 risk factors [22]) which are encouraging and suggest that combined targeted therapy plus immunotherapy may have a significant impact on survival outcomes in intermediate risk mRCC patients.

Fifty-two percent of the evaluable patients receiving AGS003 plus sunitinib demonstrated long term $(>30$ month) survival. This compares favorably with historical analysis indicating approximately $13 \%$ of intermediate and poor risk mRCC patients treated with sunitinib alone survive for $>30$ months, which has been defined as long-term in the advanced RCC setting [25]. In addition, a third of the patients on this study were alive and in follow-up or continuing treatment with AGS-003 plus sunitinib for more than 4.5 years. As of June 2014, 24\% (5/21) had survived for more than 5 years after study initiation, including 2 patients in prolonged partial remissions who continue AGS-003 booster dosing plus reduced-dose sunitinib.
The intended mechanism of action of AGS-003 is to induce $\mathrm{CD}^{+} \mathrm{CD}^{2} 8^{+} \mathrm{CD} 45 \mathrm{RA}^{-}$CTLs against patientspecific tumor antigens. Under normal conditions, activated helper $\mathrm{CD} 4^{+} \mathrm{T}$ cells up-regulate $\mathrm{CD} 40 \mathrm{~L}$ and physically interact with $\mathrm{DCs}$ resulting in effective $\mathrm{CD} 8^{+}$ cytotoxic $\mathrm{T}$ cell responses [36]. However, mRCC patients are immunosuppressed due to local and systemic influences of the tumor cells and display both DC and CD4 ${ }^{+}$ helper $\mathrm{T}$ cell dysfunction [19,37-39]. AGS-003 addresses the immune dysfunction of the host by coelectroporation of RNA encoding CD40L into the ex vivo-prepared DCs to simulate the presence of $\mathrm{CD} 4^{+}$ $\mathrm{T}$ cell help via the intracellular ligation of endogenous CD40 within the DCs. This also results in the secretion of IL-12 from the DCs, a requirement for the generation of $\mathrm{T}$ cell responses [11]. Additional functionality was engineered into AGS-003 via the novel method by which the cells are matured. Rather than employing the traditional 'cytokine cocktail' method [40], sequential cytokine exposure was employed which results in DCs that primarily induce CTL generation [10]. This functionality is important because memory $\mathrm{T}$ cell responses have been associated with good clinical outcome in patients with solid tumors [41-43]. Collectively, these properties allow AGS-003 to generate anti-tumor memory T cell responses within the immunosuppressed patient.

The increase in the absolute number of CTLs between baseline and fifth dose of AGS-003 was a statistically significant correlate to survival, even with this small number of patients (Figure $4 \mathrm{C}$ and D). The number of tumor-reactive CTLs prior to (baseline) and after the $5^{\text {th }}$ dose of AGS-003 did not correlate with survival (Figure 4A and $B$ ). This suggests that the pre-existing $T$ cells were not contributing to clinical outcome and only the newly generated, effector memory CTLs induced by AGS-003 administration were functionally significant. A positive increase in the magnitude of the induced CTL response is indicative of the induction of an antigen specific CTL response following AGS-003 administration. It is to be noted that these data were obtained prospectively since the clinical outcomes were not known at the time the immunologic assessment was carried out. The prognostic value of absolute changes in CTL numbers in response to AGS-003 as an early biomarker for overall survival is being prospectively assessed in the ongoing pivotal ADAPT Phase 3 clinical trial (Clinical Trial Registry \#NCT01582672).

In clinical practice, the most common side effects of sunitinib treatment include fatigue/asthenia, anorexia/ loss of appetite, hypothyroidism, hand-foot syndrome, stomatitis/taste changes, diarrhea/abdominal pain, myelosuppression, and hypertension [44]. The most common adverse events in the present study were consistent with those reported with sunitinib alone in advanced 
RCC. Adverse events attributed to AGS-003 were primarily mild injection-site reactions, which occurred in about half of the patients. No adverse events unique to the combination of AGS-003 plus sunitinib were reported. No grade 4 events occurred during the study and no grade 3 events considered related to AGS-003 therapy. These safety results are noteworthy, since many newly approved targeted therapies are associated with serious side effects (gastrointestinal, skin, and vascular events) that affect morbidity and mortality and limit the ability to combine these newer treatments [45].

\section{Conclusion}

When compared to outcomes and benchmarks established with targeted therapy, the addition of AGS-003 to sunitinib in an unselected, intermediate and poor risk $\mathrm{mRCC}$ patient population was associated with a doubling of expected survival, encouraging long-term and 5-year overall survival, and an excellent safety profile. In addition, the target effect of AGS-003, an expansion of effector memory CTLs, was observed after 5 doses and correlated to prolonged survival. These encouraging findings support the ongoing phase 3, randomized ADAPT study, which has been designed to compare the addition of AGS-003 with standard surgery and targeted drug therapy to standard surgery and targeted drug therapy alone in newly diagnosed, intermediate and poor risk mRCC patients.

\section{Methods}

The trial was a single-arm, open-label phase 2 study conducted from January 2008 to February 2012 at 10 centers in North America (Clinical Trial Registry \#NCT00678119). Treatment was administered according to International Conference on Harmonization Good Clinical Practice guidelines and applicable local regulatory requirements and laws, and the clinical protocol was approved by institutional review boards or independent ethics committees at each study center. All patients provided written informed consent.

\section{Patients}

Men and women at least 18 years of age with newly or recently diagnosed synchronous, metastatic RCC (mRCC) and predominantly clear cell tumor were enrolled if they had no prior nephrectomy or had a recent nephrectomy, but had at least one accessible metastatic lesion for metastasectomy. Inclusion criteria required measurable metastatic disease per Response Evaluation Criteria in Solid Tumors (RECIST) [46] and Eastern Cooperative Oncology Group (ECOG) performance status 0 or 1 . All patients included had a time from diagnosis to treatment of less than 1 year $(\mathrm{DxTx}<1 \mathrm{yr})$.

Patients were required to be candidates for sunitinib therapy and were required to have adequate end organ function. Patients with brain metastases, uncontrolled hypertension, Type 1 diabetes, active autoimmune disease, or previous systemic therapy for advanced RCC were excluded.

\section{AGS-003 Production}

AGS-003 was manufactured at a centralized GMP compliant facility (Argos Therapeutics, Durham, NC). Following screening and consent, autologous tumor total RNA was isolated from nephrectomy or metastasectomy tissue samples and messenger RNA was amplified using $\mathrm{RT} / \mathrm{PCR}$ and in vitro transcription technologies as previously described [47]. CD40L RNA was manufactured using in vitro transcription and a post-transcriptional capping method [48]. Patients had leukapheresis at the clinical site's donor center using a COBE Spectra ${ }^{\circ}$ Leukapheresis System (Gambro BCT, Lakewood, CO). Monocytes were cultured in AIM-V media with $800 \mathrm{U} /$ $\mathrm{mL}$ granulocyte macrophage-colony stimulating factor (Berlex) and $1000 \mathrm{U} / \mathrm{mL}$ IL-4 (R\&D Systems) to generate immature DCs that were then matured using $20 \mathrm{ng} / \mathrm{mL}$ tumor necrosis factor alpha (TNF $\alpha$ ) (R\&D Systems)/1000 $\mathrm{U} / \mathrm{mL} \quad$ IFN- $\gamma$ (InterMune) $/ 1 \mu \mathrm{g} / \mathrm{mL}$ prostaglandin $\mathrm{E} 2$ (Sigma). Mature DCs were electroporated with the amplified tumor RNA and CD40L RNA using a post-maturation electroporation protocol [10].

The final AGS-003 product was formulated as $1.4 \times$ $10^{7} \mathrm{DC} / 0.7 \mathrm{~mL}$ in $80 \%$ autologous plasma, $10 \%$ dextrose $(50 \% \mathrm{w} / \mathrm{v})$ (Hospira), and 10\% DMSO (Sigma) and cryopreserved in liquid nitrogen vapor phase. Thawed samples of final product were assessed for sterility, mycoplasma, endotoxin, and viability prior to release for clinical use.

\section{Treatment}

The treatment schedule is illustrated in Figure 1. Prior to initiation of AGS-003 therapy, patients initiated sunitinib therapy on a standard, repeating 6-week cycle of $50 \mathrm{mg}$ daily for 4 weeks followed by 2 week rest. Dose modifications (reductions, delays, and/or discontinuation due to toxicity) for sunitinib were permitted per standard labeling throughout study directed treatment. AGS-003 was administered prior to the initiation of the second 6-week cycle of sunitinib (week 6). Each dose of AGS-003 consisted of $1.2 \times 10^{7}$ DCs delivered as three intradermal injections of $0.2 \mathrm{~mL}(0.6 \mathrm{~mL}$ total) to the axillary lymph node basin. AGS-003 treatment continued every 3 weeks for a total of five doses (induction phase) in combination with sunitinib. Following induction, AGS-003 was administered every 12 weeks along with standard sunitinib (booster phase). Treatment was continued until disease progression, intolerable toxicity to standard of care, or end of study. Patients who continued to benefit from 
AGS-003 treatment at the time of study closure were rolled over to companion AGS-003 trials.

\section{Tumor Response Assessments}

Tumor measurements were assessed pre-nephrectomy/ metastasectomy, after one cycle of sunitinib prior to the initiation of AGS-003, and after the fifth dose of AGS-003. During booster treatment, imaging occurred every 12 weeks.

\section{Immune Response Assessments}

Preparation of blood draw samples for immune monitoring by multi-color flow cytometry. Frozen PBMCs processed by ficoll density gradient separation from whole blood draws were collected for immune monitoring prior to surgery, prior to initiation of sunitinib, and at two points after initiation of AGS-003, following the third and fifth AGS-003 dose (Figure 1). PBMCs were thawed and rested overnight in X-Vivo 15 supplemented with 10\% Human AB serum. After overnight rest PBMCs were labeled with BRDU (bromodeoxyuridine) to track T-Cell proliferation. A leukapheresis for immune monitoring was collected after the fifth dose and autologous DC targets for in vitro stimulation were prepared from DCs coelectroporated with CD40L RNA and autologous RCC tumor RNA for each evaluable subject. Autologous cultures containing DCs and PBMCs were setup and incubated at $37^{\circ} \mathrm{C}$ for 6 days. On day 6 , cultures were restimulated with autologous DCs prepared as stated above and anti-CD107a antibody was added to each tube and incubated at $37^{\circ} \mathrm{C}$ for 5 hours in the presence of Brefeldin A (BD Biosciences). After incubation, cells were stained for viability using annexin $\mathrm{V}$ and a viability dye (Invitrogen), which permits selection of viable cells, followed by surface staining with specific antibodies for detection of CD28, CD45RA, CD3, and CD8 expression. After surface staining, cells were fixed with $4 \% \mathrm{BD}$ Cytofix and stored overnight in BSA staining buffer at $4^{\circ} \mathrm{C}$. The following day, the cells were permeabilized and DNase treated for 1 hour at $37^{\circ} \mathrm{C}$ using reagents included in the BRDU staining kit (BD biosciences). Intracellular staining for IFN- $\gamma$, TNF- $\alpha$, IL-2, and BRDU were performed. After staining, cells were washed and diluted in $500 \mu \mathrm{l} \mathrm{BSA}$ buffer and transferred to a BD TruCount Tube for acquisition on a BD LSRII cytometer. 400,000600,000 events were collected per sample. Number of cells/ml was calculated using the following formula; (number of cellular events collected/number of beads collected) $\mathrm{x}$ (bead concentration)/collected volume) $\times 1000$.

\section{Gating strategy to define the absolute numbers of functional CD28+/CD45RA- CTLs}

The absolute number of cells expressing IFN-g, IL-2, TNF-a, CD107a or BRDU were first identified in the
CD8+ viable cell gate. Within each gate identifying the number of IFN-g, IL-2, TNF-a, CD107a or BRDU positive cells, the number of cells positive for $\mathrm{CD} 28$ and negative for CD45RA were subsequently determined (Figure 3 ). To determine the total number of functional CD28+/CD45RACTLs, the numbers of CD28+/CD45RA- CTLs having any function were added together to determine the absolute number of functional CD28+/CD45RA- CTLs. This was done for each subject at each indicated time point, pre and post $5^{\text {th }}$ dose AGS-003 administration. To define the overall magnitude for the response to autologous AGS-003 product the value determined pre AGS-003 administration was subtracted from the value determined after the $5^{\text {th }}$ AGS-003 dose administration to calculate the absolute change in CTLs between these two time points.

\section{Endpoints}

The efficacy and safety population consisted of all study subjects who received at least one AGS-003 dose. One patient enrolled on a previous AGS-003 monotherapy study was included in the safety, but not efficacy population because of prior AGS-003 exposure.

The primary endpoint was the CR rate as defined by RECIST v1.0 [46]. Secondary endpoints included PFS, OS, immune response and clinical benefit (CR, PR or SD).

PFS was calculated from the date of registration. The progression time of patients who did not progress at the time of final analysis or who withdrew early without documentation of progression were censored at the time of the last tumor evaluation. OS was calculated from the time of registration until death. The time of death for patients alive at the time of final analysis or lost to follow-up was censored at the last date they were known to be alive. Patients who rolled over to subsequent, companion AGS-003 trials were routinely monitored for survival.

Safety was assessed by monitoring the incidence of adverse events, laboratory assessments, localized injection-site reactions, and changes in size, tenderness, or inflammation of draining lymph nodes throughout the study. Adverse events were assessed by investigators as being possibly, probably, definitely or not related to AGS-003, sunitinib, or the combination, and graded for severity according to the NCI Common Terminology Criteria for Adverse Events (CTCAE) v3.0. Changes from baseline values in clinical laboratory tests and changes in physical examinations, vital signs, and electrocardiograms (ECGs) were determined after study drug dosing. Evaluation for autoimmune reactions was determined by assessing clinical signs and symptoms (i.e., rash, cytopenias, and arthralgias) and by laboratory assessments (i.e., anti-nuclear antibody, rheumatoid factor, anti-double stranded DNA antibody, total hemolytic complement, anti-thyroid antibody, indirect Coombs test). 


\section{Statistical Analysis}

The study was designed to enroll approximately 50 patients in order to have a sufficient number of patients who would be eligible to initiate combined AGS-003 plus sunitinib treatment and be evaluable for the assessment of primary and secondary endpoints. The planned analysis for the primary endpoint (complete response rate) required a total 38 evaluable and treated patients for $90 \%$ power to detect a CR rate of $20 \%$ versus the null rate of $5 \%$, with a type-I error of $5 \%$. No favorable risk patients were enrolled and nearly half of the evaluable patients had 3 or more RCC risk factors (i.e., poor risk) at the time of diagnosis. Study enrollment was stopped early when the sponsor concluded that the initial statistical design and expectations of complete response was inappropriate in the enrolled population of intermediate and poor risk patients. Rather than amend the protocol to a more appropriate primary endpoint such as overall survival, the decision was made to terminate further enrollment, to assess appropriate secondary endpoints, and to plan for a larger randomized study in a similar, intermediate and poor risk mRCC patient population. Prior to early termination of enrollment, twenty-five patients were recruited for study participation.

Response was assessed per RECIST v1.0, however the planned statistical analyses for the primary endpoint were not performed due to the limited enrollment to the study. PFS and OS analyses were performed using the Kaplan-Meier method with two-sided 95\% CIs for the medians. SAS version 9.2 and SPLUS version 6.2 were used for statistical analyses. Proc LIFETEST was used for Kaplan-Meier analyses. Relationship between clinical outcome and immune monitoring was analyzed using JMP version 10.0.0.

ECG, vital signs, body weight, and clinical laboratory data (actual values and changes from baseline at each assessment time point) were summarized with descriptive statistics. Laboratory results were evaluated based on laboratory-specified reference ranges and investigator determinations of clinical significance for abnormal results.

\section{Competing interest}

A. Amin, A.Z. Dudek, R.S. Lance, J.M. Holzbeierlein, J.J. Knox, V.A. Master and W. $H$. Miller report no conflict of interest/compteing interests to declare pertaining to the study; T.F. Logan reports Consulting for Argos, Bristol-Meyers Squibb, Celgene, Genentech, GlaxoSmithKline, Novartis, Pfizer, Prometheus, Wyeth, Aveo; Honoraria from Bristol-Meyers Squibb, GlaxoSmithKline, Novartis, Pfizer, Prometheus, Wyeth Research and Funding for clinical trials from Abbott, Abraxis, Argos, AstraZeneca, Aveo, Biovex, Bristol-Meyers Squibb, Celgene, Chiron, Entremed, Exelixis, GlaxoSmithKline, Immatics, Lilly, Medlmmune, Novartis, Pfizer, Roche, Schering-Plough, Synta, Wyeth, Genentech, Prometheus. S.K. Pal reports Consulting for Novartis, Aveo and Honoraria (Speaker's Bureau) from Pfizer, Novartis, Astellas.

L.I. Karsh reports Consulting for Amgen, Algeta, Astellas, Aveo, Bayer

Dendreon, Janssen, Medivation, Spectrum, and Swan Valley Medical, was a Speaker for Amgen, Astellas, Dendreon, GSK, Janssen, and Medivation, and received clinical trial funding from Amgen, Argos, FKD, Telomedix, Astellas,
Dendreon, Janssen, Medivation, Spectrum ,SWOG, RTOG,Nymox. R.A. Figlin reports Research Funding from Argos, BMS, GSK, Immatics, Novartis; and Consulting/Scientific Advisory Board member for Galena, Pfizer, Onyx. M.A. DeBenedette, I.Y. Tcherepanova, W.L. Williams, D.C. Plessinger and C.A. Nicolette are Argos employees.

\section{Author contributions}

All authors participated in manuscript writing, review and revision. In addition, AA participated in study conception and design, method development, data acquisition, analysis and interpretation and study supervision; AZD, TFL, RSL, JMH, JJK, SKP, WHM, and LIK participated in study conception and design, method development, data acquisition, and study supervision; VAM participated in study conception and design, method development, and study supervision; IYT participated in data analysis and interpretation and technical and materials support; MADeB participated in data acquisition, analysis and interpretation and technical and materials support; WLW participated in data analysis and interpretation and study administration; DCP participated in data analysis and interpretation, study administration and study supervision; CAN participated in study conception and design, method development, data analysis and interpretation and study administration; RAF participated in data analysis and interpretation, and study supervision. All authors read and approved the final manuscript.

\section{Acknowledgments}

Patrice C. Ferriola provided editorial assistance in the preparation for the manuscript and was supported by Argos Therapeutics, Inc.

\section{Author details}

${ }^{1}$ Levine Cancer Institute, Charlotte, NC, USA. ${ }^{2}$ University of Illinois Cancer Center, Chicago, IL, USA. ${ }^{3}$ Indiana University Simon Cancer Center, Indianapolis, IN, USA. ${ }^{4}$ Urology of Virginia, Norfolk, VA, USA. ${ }^{5}$ University of Kansas Medical Center, Kansas City, KS, USA. ${ }^{6}$ Princess Margaret Hospital, Toronto, ON, Canada. ${ }^{7}$ Emory University, Atlanta, GA, USA. ${ }^{8}$ City of Hope Comprehensive Cancer Center, Duarte, CA, USA. ${ }^{9}$ Lady Davis Institute and Segal Cancer Center-Jewish General Hospital, McGill University, Montreal, QC, Canada. ${ }^{10}$ The Urology Center of Colorado, Denver, CO, USA. ${ }^{11}$ Argos Therapeutics, Inc., Durham, NC, USA. ${ }^{12}$ Cedars-Sinai Medical Center, Los Angeles, CA, USA

Received: 6 October 2014 Accepted: 2 March 2015

Published online: 21 April 2015

\section{References}

1. Singer EA, Gupta GN, Srinivasan R. Targeted therapeutic strategies for the management of renal cell carcinoma. Curr Opin Oncol. 2012;24(3):284-90.

2. Coppin C, Kollmannsberger C, Le L, Porzsolt F, Wilt TJ. Targeted therapy for advanced renal cell cancer (RCC): a Cochrane systematic review of published randomised trials. BJU Int. 2011;108(10):1556-63.

3. Albiges L, Oudard S, Negrier S, Caty A, Gravis G, Joly F, et al. Complete remission with tyrosine kinase inhibitors in renal cell carcinoma. J Clin Oncol. 2012;30(5):482-7.

4. Gore ME, Larkin JM. Challenges and opportunities for converting renal cell carcinoma into a chronic disease with targeted therapies. Br J Cancer. 2011;104(3):399-406

5. George S, Pili R, Carducci MA, Kim JJ. Role of immunotherapy for renal cell cancer in 2011. J Natl Compr Canc Netw. 2011:9(9):1011-8.

6. Atkins MB. Treatment selection for patients with metastatic renal cell carcinoma: identification of features favoring upfront IL-2-based immunotherapy. Med Oncol. 2009;26 Suppl 1:18-22.

7. Banchereau J, Paczesny S, Blanco P, Bennett L, Pascual V, Fay J, et al. Dendritic cells: controllers of the immune system and a new promise for immunotherapy. Ann N Y Acad Sci. 2003;987:180-7.

8. Tacken P, De Vries I, Torensma R, Figdor C, Tacken PJ, De Vries IJM, et al. Dendritic-cell immunotherapy: from ex vivo loading to in vivo targeting. Nat Rev Immunol. 2007;10:790-802.

9. Calderhead D, Hedgepeth A, Ketteringham $H$, Horvatinovich J, Nicolette C, Healey D. Development of CD4+ independent CD8+ CTL activity in vitro by transfection of CD154 (CD40 ligand) mRNA into dendritic cells. Keystone Symposia on Molecular and Cellular Biology. February 1-7, 2005. Vancouver, BC. Poster presentation. 
10. Calderhead DM, DeBenedette MA, Ketteringham H, Gamble AH, Horvatinovich JM, Tcherepanova IY, et al. Cytokine maturation followed by CD40L mRNA electroporation results in a clinically relevant dendritic cell product capable of inducing a potent proinflammatory $C T L$ response. J Immunother. 2008;31(8):731-41.

11. DeBenedette MA, Calderhead DM, Ketteringham H, Gamble AH, Horvatinovich JM, Tcherepanova IY, et al. Priming of a novel subset of CD28+ rapidly expanding high-avidity effector memory CTL by post maturation electroporation-CD40L dendritic cells is IL-12 dependent. J Immunol. 2008;181(8):5296-305.

12. DeBenedette MA, Calderhead DM, Tcherepanova IY, Nicolette CA, Healey DG. Potency of mature CD40L RNA electroporated dendritic cells correlates with IL-12 secretion by tracking multifunctional CD8(+)/CD28(+) cytotoxic T-cell responses in vitro. J Immunother. 2011;34(1):45-57.

13. Logan TF, Gardner TA, Amin A, Cohen V, Wong MKK, Master VA, et al. Phase 1/2 Study of AGS-003, a personalized immunotherapeutic evaluated in newly diagnosed metastatic renal cell carcinoma subjects. J Clin Oncol. 2010:28 (suppl; abstr 379).

14. Heiser A, Dahm P, Yancey DR, Maurice MA, Boczkowski D, Nair SK, et al. Human dendritic cells transfected with RNA encoding prostate-specific antigen stimulate prostate-specific CTL responses in vitro. J Immunol. 2000;164(10):5508-14.

15. Mitchell DA, Nair SK. RNA transfected dendritic cells as cancer vaccines. Curr Opin Mol Ther. 2000;2(2):176-81.

16. Mitchell DA, Nair SK. RNA-transfected dendritic cells in cancer immunotherapy. J Clin Invest. 2000;106(9):1065-9.

17. Dannull J, Su Z, Rizzieri D, Yang BK, Coleman D, Yancey D, et al. Enhancement of vaccine-mediated antitumor immunity in cancer patients after depletion of regulatory T cells. J Clin Invest. 2005;115(12):3623-33.

18. Ko JS, Zea AH, Rini Bl, Ireland $J$, Elson P, Cohen P, et al. Sunitinib mediates reversal of myeloid-derived suppressor cell accumulation in renal cell carcinoma patients. Clin Cancer Res. 2009;15(6):2148-57.

19. Finke JH, Rini B, Ireland J, Rayman P, Richmond A, Golshayan A, et al. Sunitinib reverses type-1 immune suppression and decreases T-regulatory cells in renal cell carcinoma patients. Clin Cancer Res. 2008;14(20):6674-82.

20. Vieweg J, Su Z, Dahm P, Kusmartsev S. Reversal of tumor-mediated immunosuppression. Clin Cancer Res. 2007;13(2 Pt 2):727s-32s.

21. Kusmartsev S, Su Z, Heiser A, Dannull J, Eruslanov E, Kubler H, et al. Reversal of myeloid cell-mediated immunosuppression in patients with metastatic renal cell carcinoma. Clin Cancer Res. 2008;14(24):8270-8.

22. Ko JJ, Choueiri TK, Rini Bl, Lee JL, Kroeger N, Srinivas S, et al. First-, second-, third-line therapy for mRCC: benchmarks for trial design from the IMDC. Br J Cancer. 2014:110(8):1917-22.

23. Motzer RJ, Escudier B, Bukowski R, Rini Bl, Hutson TE, Barrios CH, et al. Prognostic factors for survival in 1059 patients treated with sunitinib for metastatic renal cell carcinoma. Br J Cancer. 2013;108(12):2470-7.

24. Motzer RJ, Bacik J, Murphy BA, Russo P, Mazumdar M. Interferon-alfa as a comparative treatment for clinical trials of new therapies against advanced renal cell carcinoma. J Clin Oncol. 2002;20(1):289-96.

25. Heng DY, Xie W, Regan MM, Warren MA, Golshayan AR, Sahi C, et al. Prognostic factors for overall survival in patients with metastatic renal cell carcinoma treated with vascular endothelial growth factor-targeted agents: results from a large, multicenter study. J Clin Oncol. 2009;27(34):5794-9.

26. Motzer RJ, Hutson TE, Tomczak P, Michaelson MD, Bukowski RM, Rixe O, et al. Sunitinib versus interferon alfa in metastatic renal-cell carcinoma. N Engl J Med. 2007;356(2):115-24.

27. Rini Bl, Bellmunt J, Clancy J, Wang K, Niethammer AG, Hariharan S, et al. Randomized phase III trial of temsirolimus and bevacizumab versus interferon alfa and bevacizumab in metastatic renal cell carcinoma: INTORACT trial. J Clin Oncol. 2014;32(8):752-9.

28. Patel PH, Senico PL, Curiel RE, Motzer RJ. Phase I study combining treatment with temsirolimus and sunitinib malate in patients with advanced renal cell carcinoma. Clin Genitourin Cancer. 2009;7(1):24-7.

29. Molina AM, Feldman DR, Voss MH, Ginsberg MS, Baum MS, Brocks DR, et al. Phase 1 trial of everolimus plus sunitinib in patients with metastatic renal cell carcinoma. Cancer. 2012;118(7):1868-76.

30. Feldman DR, Baum MS, Ginsberg MS, Hassoun H, Flombaum CD, Velasco S, et al. Phase I trial of bevacizumab plus escalated doses of sunitinib in patients with metastatic renal cell carcinoma. J Clin Oncol. 2009;27(9):1432-9.

31. Motzer RJ, Hutson TE, Tomczak P, Michaelson MD, Bukowski RM, Oudard S, et al. Overall survival and updated results for sunitinib compared with interferon alfa in patients with metastatic renal cell carcinoma. J Clin Oncol. 2009:27(22):3584-90.

32. Motzer RJ, Hutson TE, Olsen MR, Hudes GR, Burke JM, Edenfield WJ, et al. Randomized phase II trial of sunitinib on an intermittent versus continuous dosing schedule as first-line therapy for advanced renal cell carcinoma. J Clin Oncol. 2012;30(12):1371-7.

33. Nishino M, Jagannathan JP, Krajewski KM, O'Regan K, Hatabu H, Shapiro G, et al. Personalized tumor response assessment in the era of molecular medicine: cancer-specific and therapy-specific response criteria to complement pitfalls of RECIST. AJR Am J Roentgenol. 2012;198(4):737-45.

34. Hodi FS, O'Day SJ, McDermott DF, Weber RW, Sosman JA, Haanen JB, et al. Improved survival with ipilimumab in patients with metastatic melanoma. N Engl J Med. 2010;363(8):711-23.

35. Lebbe C, Weber JS, Maio M, Neyns B, Harmankaya K, Hamid O, et al. Survival follow-up and ipilimumab retreatment for patients with advanced melanoma who received ipilimumab in prior phase II studies. Ann Oncol 2014, Sep 10. [Epub ahead of print].

36. Feau S, Arens R, Togher S, Schoenberger SP. Autocrine IL-2 is required for secondary population expansion of CD8(+) memory T cells. Nat Immunol. 2011;12(9):908-13.

37. Porta C, Bonomi L, Lillaz B, Paglino C, Rovati B, Imarisio I, et al. Renal cell carcinoma-induced immunosuppression: an immunophenotypic study of lymphocyte subpopulations and circulating dendritic cells. Anticancer Res. 2007;27(1A):165-73.

38. Ma Y, Shurin GV, Peiyuan Z, Shurin MR. Dendritic cells in the cancer microenvironment. J Cancer. 2013;4(1):36-44.

39. Knutson $\mathrm{KL}$, Disis ML, Salazar LG. CD4 regulatory $T$ cells in human cancer pathogenesis. Cancer Immunol Immunother. 2007:56(3):271-85.

40. Lee AW, Truong T, Bickham K, Fonteneau JF, Larsson M, Da Silva I, et al. A clinical grade cocktail of cytokines and PGE2 results in uniform maturation of human monocyte-derived dendritic cells: implications for immunotherapy. Vaccine. 2002;20 Suppl 4:A8-A22.

41. Pages F, Kirilovsky A, Mlecnik B, Asslaber M, Tosolini M, Bindea G, et al. In situ cytotoxic and memory $T$ cells predict outcome in patients with earlystage colorectal cancer. J Clin Oncol. 2009;27(35):5944-51.

42. Perret $\mathrm{R}$, Ronchese F. Memory T cells in cancer immunotherapy: which CD8 T-cell population provides the best protection against tumours? Tissue Antigens. 2008;72(3):187-94.

43. Klebanoff CA, Gattinoni L, Restifo NP. CD8+ T-cell memory in tumor immunology and immunotherapy. Immunol Rev. 2006;211:214-24.

44. Kollmannsberger C, Bjarnason G, Burnett P, Creel P, Davis M, Dawson N, et al. Sunitinib in metastatic renal cell carcinoma: recommendations for management of noncardiovascular toxicities. Oncologist. 2011;16(5):543-53.

45. Niraula S, Seruga B, Ocana A, Shao T, Goldstein R, Tannock IF, et al. The price we pay for progress: A meta-analysis of harms of newly approved anticancer drugs. J Clin Oncol. 2012;30:3012-9.

46. Therasse P, Arbuck SG, Eisenhauer EA, Wanders J, Kaplan RS, Rubinstein L, et al. New guidelines to evaluate the response to treatment in solid tumors. European Organization for Research and Treatment of Cancer, National Cancer Institute of the United States, National Cancer Institute of Canada. J Natl Cancer Inst. 2000;92(3):205-16.

47. Slagter-Jager JG, Raney A, Lewis WE, Debenedette MA, Nicolette CA, Tcherepanova IY. Evaluation of RNA Amplification Methods to Improve DC Immunotherapy Antigen Presentation and Immune Response. Mol Ther Nucleic Acids. 2013;2:e91.

48. Tcherepanova I, Harris J, Starr A, Cleveland J, Ketteringham H, Calderhead D, et al. Multiplex RT-PCR amplification of HIV genes to create a completely autologous DC-based immunotherapy for the treatment of HIV infection. PLoS One. 2008;3(1):e1489. 